\title{
Probing the Phase Separation in the Doped Manganites by the Magnetic Resonance Methods
}

\author{
N. Volkov*, G. Petrakovskit, K. Sablina and K. Patrin \\ L.V. Kirensky Institute of Physics SB RAS, Krasnoyarsk, 660036, Russia
}

Here we present magnetic resonance study which confirms an existence of a phase separation picture in $\mathrm{Eu}_{0.7} \mathrm{~Pb}_{0.3} \mathrm{MnO}_{3}$ single crystals. The measurements were performed with a standard technique and with using unconventional schemes of the magnetic resonance method. The inhomogeneous paramagnetic-ferromagnetic state, which is sensitive to a magnetic field, takes place in $0.65 T_{\mathrm{c}}-1.15 T_{\mathrm{c}}$ temperature range, where the colossal magnetoresistance effect is observed. The behavior of spectra suggests that a scenario of the phase separation is realized and the mixed state is not related to a simple chemical inhomogeneity. The changes of the conductivity induced by the microwave resonance absorption are observed in a vicinity of $T_{c}$, too. The changes are not related to a trivial heating of the sample by a microwave radiation. We propose a mechanism connected with the change of equilibrium concentrations of the coexisting phases, this change takes place when the system is under magnetic resonance conditions. To analyze the experimental data we applied the effective-medium approximation for two-component system. Also we attracted the simplest phenomenological model qualitatively reproducing the essential features of the behavior of the two-phase system.

PACS numbers: $76.50 .+\mathrm{g}, 72.20 . \mathrm{My}$

\section{Introduction}

At present a variety of experimental studies support the existence of the inhomogeneous states near the ferromagnetic ordering temperature $T_{\mathrm{c}}$ in some parts of the manganite phase diagram [1]. These inhomogeneities are generally described in the framework of the phase separation picture, when the coexistence of a metallic/ferromagnetic and insulating/paramagnetic (or antiferromagnetic)

*corresponding author; e-mail: volk@iph.krasn.ru 
phases take place in the material. Such mixed state is thermodynamic equilibrium state and one is not related to simple chemical inhomogeneity of the samples. Full understanding of the phase separation phenomenon in the manganites is still lacking. At the same time, it is evident that phase separation is due to competition between a variety of interactions with comparable energy scales [2].

The phase separation scenarios discussed at present for manganites suppose the different magnetic state of the coexisting phases, so the electron magnetic resonance method can be an effective tool for probing the phase separation in the samples. Really, works carried out by several research groups with using magnetic resonance technique in a wide temperature range show that the magnetic mixed-phase state is realized in the vicinity of $T_{\mathrm{c}}[3-6]$. But all studies, as a rule, were carried out with using conventional schemes of the magnetic resonance method.

In this work we used the magnetic resonance methods for probing the inhomogeneity of the $\mathrm{Eu}_{0.7} \mathrm{~Pb}_{0.3} \mathrm{MnO}_{3}$ single crystal. At that, measurements were performed with both standard and unconventional technique. The similar results were obtained early for $\mathrm{La}_{0.7} \mathrm{~Pb}_{0.3} \mathrm{MnO}_{3}[7]$.

\section{Experimental}

The single crystals of the $\mathrm{Eu}_{0.7} \mathrm{~Pb}_{0.3} \mathrm{MnO}_{3}$ were grown by a method of spontaneous crystallization from solution in a melt. The choice of the $\mathrm{Pb}$ ions was dictated by the peculiarity of the technology, the mixture of $\mathrm{PbO}$ and $\mathrm{PbF}_{2}$ was used as the solvent, and at the same time it provided the lead content in the crystals. X-ray analyses confirmed the composition of the crystals and showed that the samples are single-phase perovskites with rhombohedral $(R \overline{3} c)$ structure. The samples have the temperature of the ferromagnetic ordering $T_{\mathrm{c}}=210 \mathrm{~K}$. All measurements were carried out on well polished plate-like samples of the size of about $2 \times 2 \times 0.1 \mathrm{~mm}^{3}$.

For the magnetic resonance measurements we used a standard cavity perturbation technique with microwave frequency $\nu=25 \mathrm{GHz}$ and a spectrometer operating in $\nu=37-80 \mathrm{GHz}$ frequency range with a pulsed external magnetic field up to $40 \mathrm{kOe}$. Moreover, the spectrometer with combined influence of the microwave irradiation and direct current was used to study a change of the conductivity induced by the magnetic resonance microwave absorption. In this case two leads were attached to the sample by the silver epoxy. To detect a change of the conductivity a dc voltage on the sample was measured under the magnetic resonance, at the same time the current through sample was constant.

The magnetization data were obtained by SQUID magnetometer. The magnetoresistance was measured by the standard four-probe technique with external magnetic field up to $12 \mathrm{kOe}$. 


\section{Experimental results}

We presented the features of the magnetic resonance spectra for the $\mathrm{Eu}_{0.7} \mathrm{~Pb}_{0.3} \mathrm{MnO}_{3}$ single crystal in part in a previous paper [8]. At first we used conventional scheme of the spectrometer operating at $25 \mathrm{GHz}$ with field modulation. It was observed that the spectra reveal a structure in temperature range from $240 \mathrm{~K}$ to $140 \mathrm{~K}$. We applied a fitting procedure by Lorentzian lines, which showed that the observed spectra could be fitted satisfactorily, only if we assumed the presence of two lines. Figure 1a shows the temperature dependences of the integrated intensities $I_{\mathrm{pm}}$ and $I_{\mathrm{fm}}$ of two coexisting lines in the spectra. We suppose that two lines in the spectra are the magnetic resonance absorption from paramagnetic (PM) and ferromagnetic (FM) regions in the sample (the confirmation for this will be given below). The line corresponding to ferromagnetic resonance absorption in the spectra appears at the temperature $T \approx 235 \mathrm{~K}$, which is above $T_{\mathrm{c}}$. It is interesting to notice that for temperatures below $235 \mathrm{~K}$ the curve for the inverse dc susceptibility $1 / \chi_{\mathrm{dc}}$ versus temperature shows the positive curvature suggesting spin clustering effects. When the temperature approaches $T_{\mathrm{c}}$ from above the intensity of the PM resonance line $I_{\mathrm{pm}}$ follows reasonably well the temperature dependence of a PM susceptibility $\chi_{\mathrm{dc}}$, which strongly increases. Comparing the dependences for $I_{\mathrm{pm}}$ and $I_{\mathrm{fm}}$ with temperature behavior of the magnetoresistance $\Delta \rho / \rho_{0}$, one can see that colossal magnetoresistance (CMR) effect is observed in temperature range where PM and FM regions coexist in the sample. Let us note, however, that not only the phase volumes make contribution to the line intensities. Therefore above we concluded that $I_{\mathrm{pm}}$ is proportional to $\chi_{\mathrm{dc}}$, and we can suppose that $I_{\mathrm{fm}}$ is proportional to $M_{\mathrm{fm}}(H, T)$.

Figure 1b shows the temperature dependences of the resonance fields ( $H_{\mathrm{r}}^{\mathrm{fm}}$ and $\left.H_{\mathrm{r}}^{\mathrm{pm}}\right)$ and line widths $\left(\Delta H_{\mathrm{fm}}\right.$ and $\left.\Delta H_{\mathrm{pm}}\right)$ for the FM and PM absorption lines in the magnetic resonance spectra. We discussed earlier the questions concerning a great broadening of the $\Delta H_{\mathrm{fm}}$ and $\Delta H_{\mathrm{pm}}$ below $T_{\mathrm{c}}[8]$.

The shift of the resonance field to lower fields for absorption line corresponding to the FM phase $H_{\mathrm{r}}^{\mathrm{fm}}$ is attributed to increase in magnetization of the sample. Our samples may be approximated by infinite plate and so the expression for $H_{\mathrm{r}}^{\mathrm{fm}}$ can be written as

$$
\frac{\omega}{\gamma}=\left[H_{\mathrm{r}}^{\mathrm{fm}}\left(H_{\mathrm{r}}^{\mathrm{fm}}+4 \pi M_{\mathrm{fm}}^{*}\right)\right]^{1 / 2}
$$

here, $\omega=2 \pi \nu$ is a cyclic frequency, $\gamma$ is a gyromagnetic ratio, $M_{\mathrm{fm}}^{*}$ is an effective magnetization, which may differ from $M_{\mathrm{fm}}$ due to influence of the magnetic anisotropy and the magnetic inhomogeneity of the crystal. The magnetic anisotropy of the 3D perovskite-like manganites is generally small, in our case one is no more than 120 Oe at $T=100 \mathrm{~K}$.

The PM line shifts to lower field as $T$ decreases from $T_{c}$ can be explained either by the influence of the demagnetizing fields from the FM regions or by the change of the $g$-factor which takes place as a result of the Jahn-Teller distortions 


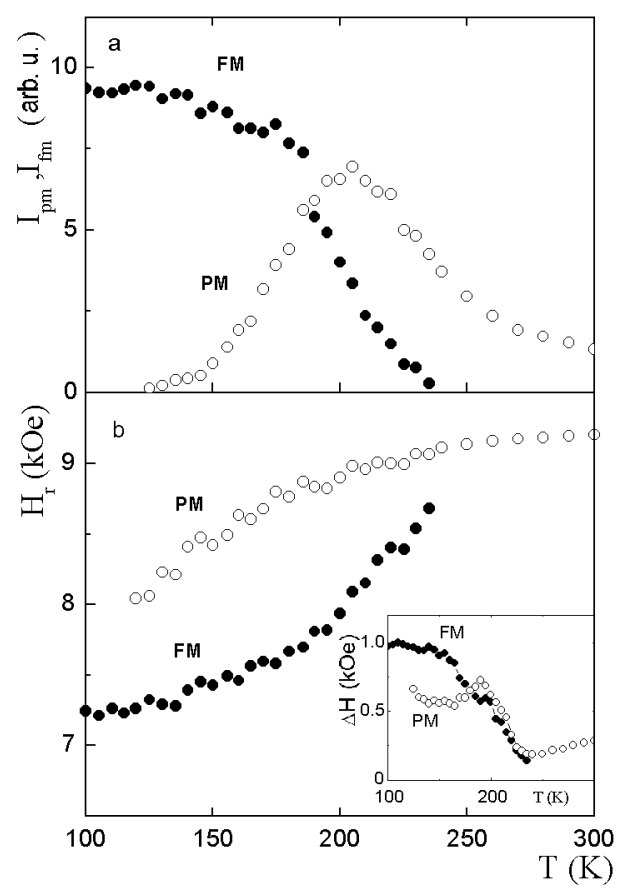

Fig. 1. Temperature dependences of the paramagnetic and ferromagnetic line parameters $(\nu=25 \mathrm{GHz})$ : (a) intensity, (b) resonance field, and (inset) line width.

at $T<T_{\mathrm{c}}$. It should be noted that in the case of $\mathrm{La}_{0.7} \mathrm{~Pb}_{0.3} \mathrm{MnO}_{3}$ [7], we did not observe the shift of the resonance field of PM line with the exception of small increase in one at $T \sim T_{c}$.

The study of the field-frequency dependences of the magnetic resonance spectra is found to be a very powerful method to determine the magnetic state of the system and its behavior in the external magnetic field. At first we have applied this method for the study of the mixed two-phase state in the $\mathrm{La}_{0.7} \mathrm{~Pb}_{0.3} \mathrm{MnO}_{3}$ single crystal [7]. Figure 2 shows the field-frequency dependences for two observed lines in the magnetic resonance spectra of the $\mathrm{Eu}_{0.7} \mathrm{~Pb}_{0.3} \mathrm{MnO}_{3}$ at $T=205 \mathrm{~K}$. It is expected that the dependence for FM absorption line will follow the expression (1). Since at $T=205 \mathrm{~K}$ the sample is far from the magnetic saturation we must take into consideration the dependence of $M_{\mathrm{fm}}^{*}$ on the temperature. To fit the experimental data we supposed that $M_{\mathrm{fm}}^{*}(H)$ and $M_{\mathrm{fm}}(H)$ do not strongly differ from each other, and the Brillouin function for the $M_{\mathrm{fm}}(H)$ was used

$$
M_{\mathrm{fm}}=M_{0} B_{S}\left\{\frac{3 T_{\mathrm{c}} S}{k_{\mathrm{B}} T}\left[\frac{g \mu_{\mathrm{B}}(S+1)}{3 k_{\mathrm{B}} T_{\mathrm{c}}} H+\frac{M_{\mathrm{fm}}}{M_{0}}\right]\right\},
$$

here, $M_{0}$ is the saturation magnetization, $S=2(1-x)+3 x / 2$ and $x=0.3$. The result of fitting is shown in Fig. 2 by dotted line for $M_{\mathrm{fm}}(H, T)=0.15$ at $T=205 \mathrm{~K}$ and $H=0$. 


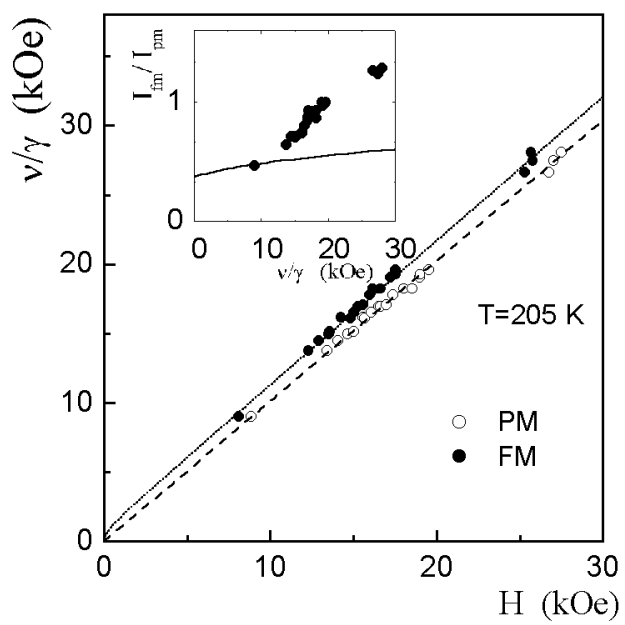

Fig. 2. Frequency-field dependences of the paramagnetic and ferromagnetic absorption lines observed in the magnetic resonance spectrum at $205 \mathrm{~K}$. Dotted lines are extrapolations of experimental points by the dependences (1) and (3) (see the text). Inset: the ratio of the intensities of the ferromagnetic and paramagnetic lines $\left(I_{\mathrm{fm}} / I_{\mathrm{pm}}\right)$ versus microwave frequency; solid line represents the expected dependence under the assumption that $I_{\mathrm{fm}}$ depends only on $M_{\mathrm{fm}}(H)$.

For the PM regions the demagnetizing effect can be neglected as the magnetization is small. It turned out that the experimental data are well fitted by the following equation:

$$
\frac{\omega}{\gamma}=H_{\mathrm{r}}^{\mathrm{pm}}+H_{\mathrm{eff}}
$$

where the external magnetic field $H$ for the PM regions is corrected by the effective field $H_{\mathrm{eff}}$. The dependence of the $H_{\mathrm{eff}}$ on $H$ has behavior similar to $M_{\mathrm{fm}}(H)$ (see Eq. (2)), which has been used for approximation of the frequency-field dependence for $\mathrm{FM}$ line. The value of the $H_{\mathrm{eff}}$ amounts to $\approx 15 \%$ of the $4 \pi M_{\mathrm{fm}}(H)$ value. This suggests such kind of the mixed-phase picture in the crystal, where the PM regions are located in the demagnetizing field from FM regions. Further support for this conclusion is the temperature dependence of the $H_{\mathrm{r}}^{\mathrm{pm}}$ below $T_{\mathrm{c}}$ (see Fig. $1 \mathrm{~b}$ ). With decreasing temperature, the magnetization and volume of the FM phase in the sample grow, which leads to the increase in the demagnetizing field. As a result, the resonance field $H_{\mathrm{r}}^{\mathrm{pm}}$ shifts to the low fields.

It is interesting to notice that with microwave frequency (or the magnetic field) increasing the relative intensity of the FM line $I_{\mathrm{fm}}$ increases; on the contrary, PM line intensity $I_{\mathrm{pm}}$ decreases. This result is shown in the inset of Fig. 2, where the ratio $I_{\mathrm{fm}} / I_{\mathrm{pm}}$ as a function of microwave frequency is plotted. The observed behavior of the $I_{\mathrm{fm}} / I_{\mathrm{pm}}$ cannot be explained only by the increase in $M_{\mathrm{fm}}(H)$ [8]. This observation suggests that the magnetic field changes the phase state of the 
sample, as $H$ increases, the volume of FM phase in the sample grows but the volume of the PM phase is reduced. This mechanism leads to observed growth of the ratio $\left(I_{\mathrm{fm}} / I_{\mathrm{pm}}\right)$. One can notice that the above results confirm the picture of the phase separation in the sample because the strong effect of the external magnetic field can hardly be expected in the case when the chemical inhomogeneity of the sample takes place.

Now we analyze a one more unexpected effect which is the change of the sample conductivity induced by the magnetic resonance absorption. It is interesting that such change is observed only in temperature range where separation on PM and FM phase takes place in the sample. In Fig. 3a the dependences of the magnetoresistance $\Delta \rho / \rho_{0}$ on magnetic field both with microwave irradiation and without it are presented. Also in this picture one can see the change of the magnetoresistance which takes place only due to resonance microwave absorption. These data were obtained for $T=195 \mathrm{~K}$, where the effect has the maximal value. The coexistence of the PM and FM resonance lines is observed in magnetic resonance spectrum. Figure $3 \mathrm{~b}$ shows the relative resonance spectrum. The dependence of the resistance $R$ on magnetic field under magnetic resonance has dispersion-like

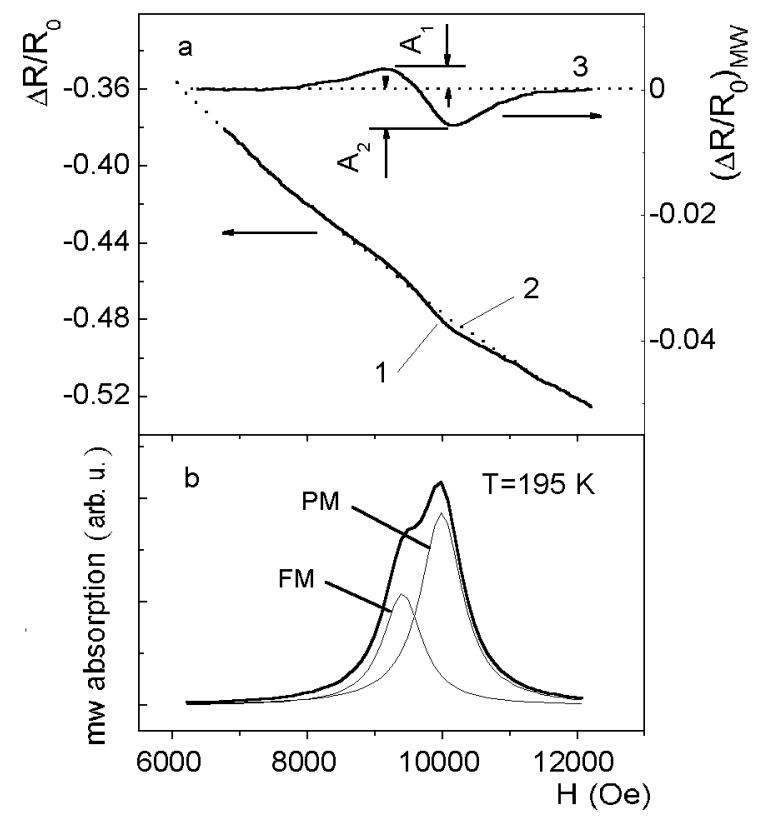

Fig. 3. (a) The dependences of the magnetoresistance $\Delta \rho / \rho_{0}$ on magnetic field: 1 under magnetic resonance absorption; 2 - without microwave irradiation; 3 - the change of the magnetoresistance which takes place only due to resonance microwave absorption. (b) The magnetic resonance spectrum and the best fitting of the spectrum by two-Lorentzian lines. 
shape. Increase in $R$ corresponds to a region of the magnetic fields where FM resonance absorption is observed and decreasing $R$ takes place under PM absorption. Such behavior suggests that heating mechanism cannot be responsible for this effect. The relative change of resistivity $\Delta \rho / \rho_{0}$ induced by resonance microwave absorption has a linear dependence on the microwave power $P$ and reaches $0.2 \%$ at $P \approx 100 \mathrm{~mW}$. The amplitude of the change depends on a value of the dc-current $I$ through the sample and exhibits saturation at $I>1 \mathrm{~mA}$, Fig. 4 . At the same time, a $V-I$ characteristic is linear over all current range up to $1 \mathrm{~mA}$, see the inset in Fig. 4. Figure 5 shows the temperature behavior of the changes of the sample resistivity induced by the magnetic resonance. We can see a correlation of the temperature dependences for this effect and the magnetoresistance (the inset

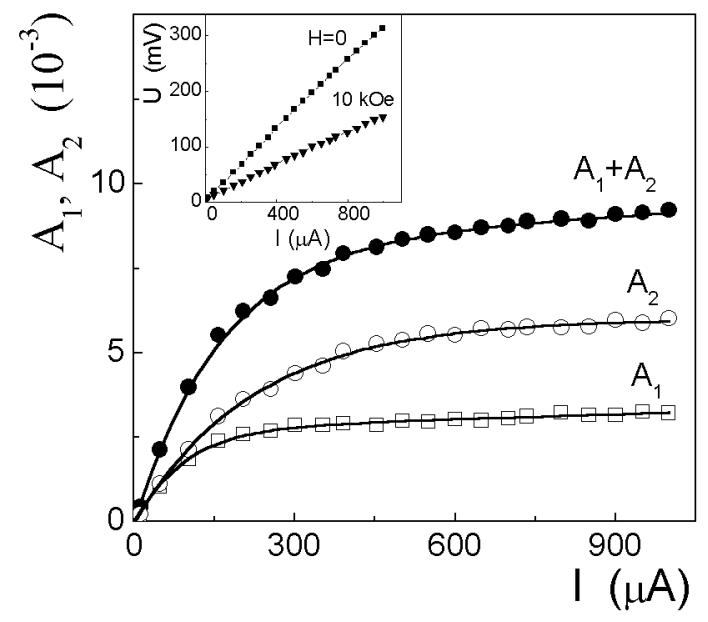

Fig. 4. The dependences of amplitudes of the $A_{1}$ and $A_{2}$ signals (see Fig. 2) on a value of the dc current at $T=195 \mathrm{~K}$. Inset: voltage-current characteristic.

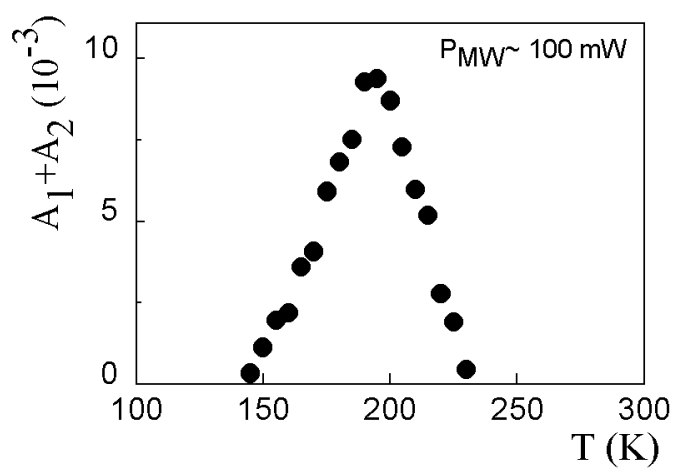

Fig. 5. The temperature dependence of amplitude of the $A_{1}$ and $A_{2}$ signals (see Fig. 2), the power of the microwave irradiation is $100 \mathrm{~mW}$. 
in Fig. 1). Presented experimental data suggest that state with the phase separation in the sample plays a key role in a mechanism which invokes the changes of the conductivity under the magnetic resonance.

\section{Effective-medium approximation for two-phase composite}

We have analyzed the experimental data in the framework of the effective-medium approximation. This approach implies that the observed electrical resistivity arises from a field- and temperature-dependent concentration of the metallic regions within semiconductive background with polaronic conductivity.

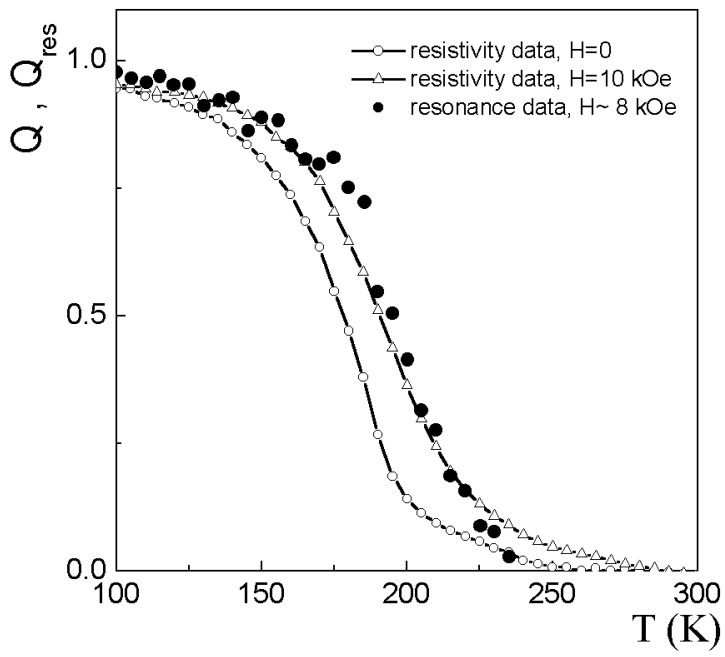

Fig. 6. The temperature dependences of concentration of the ferromagnetic phase in the sample. Open circles and triangles represent the dependences calculated in the framework of the effective-medium approximation for magnetic field $10 \mathrm{kOe}$ and without magnetic field. Filled circles are the same dependence obtained from magnetic resonance data (see the text).

For obtaining the dependences of the resistivity on the temperature for the metallic and semiconductive regions we approximate the experimental resistivity data at $T<120 \mathrm{~K}$ and $T>250 \mathrm{~K}$, respectively. In these temperature ranges the sample is certainly in homogeneous state. The data for metallic state, below $120 \mathrm{~K}$, are well fitted by the power law

$$
\rho_{\mathrm{fm}}(T)=7.6 \times 10^{-2}+1.3 \times 10^{-4} T^{2}+1.3 \times 10^{-12} T^{5}[\Omega \mathrm{cm}] .
$$

Here the terms with $T^{2}$ and $T^{5}$ correspond to contributions from one-magnon and electron-phonon scattering processes [9]. Above $250 \mathrm{~K}$, where only the paramagnetic state takes place, the resistivity can be given by the form expected for small polaronic mechanism 


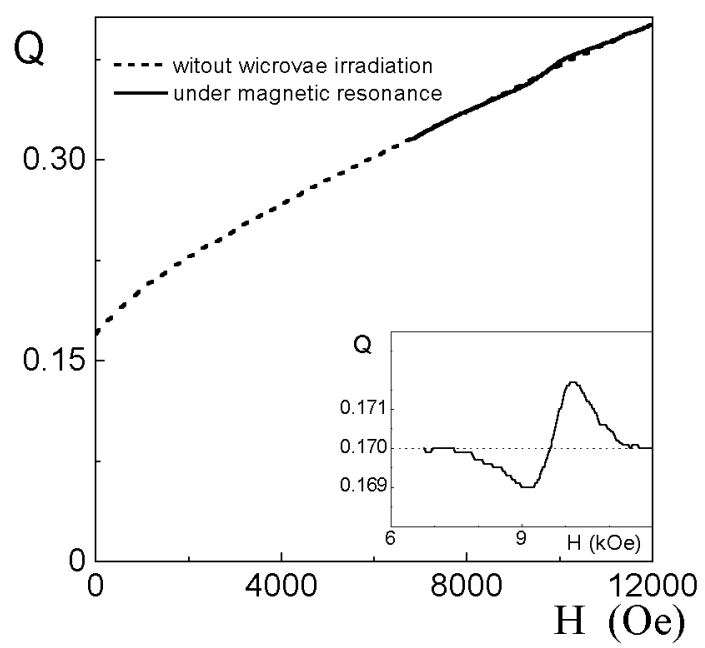

Fig. 7. The magnetic field dependences of concentration of the ferromagnetic phase in the sample. The dependences were calculated in the framework of the effective-medium approximation for the experimental data without microwave irradiation and under magnetic resonance, $T=195 \mathrm{~K}$. Inset: the change of concentration of ferromagnetic phase which is needed to explain the change of the conductivity under magnetic resonance.

$$
\rho_{\mathrm{pm}}(T)=1.6 \times 10^{-3} T \exp \left(\frac{1145}{T}\right)[\Omega \mathrm{cm}]
$$

Then we assume that $\rho=\rho(H, T)$ in the transition region satisfies the general expression for the effective resistivity of the composite consisting of ellipsoidal metallic regions in the semiconducting matrix [10]. This approach allows us to determine the concentration of the metallic phase as function of the magnetic field and temperature $Q=Q(H, T)$. Results are shown in Figs. 6 and 7. In Fig. 6 we also plotted the normalized value $Q_{\text {res }}=I_{\mathrm{fm}} / M(H, T)$ obtained from an analysis of the magnetic resonance data. This value corresponds to the volume of the ferromagnetic regions in the sample. On condition that $F M$ regions are in the metallic state the $Q_{\text {res }}$ and $Q$ should have the identical dependences. Really, Fig. 5 shows the satisfactory coincidence for temperature dependences $Q_{\text {res }}$ and $Q$ obtained in two independent ways. Figure 7 shows the dependences $Q$ both without microwave irradiation and under the magnetic resonance. The change of $Q$ which is needed to account for the change of the conductivity induced by the magnetic resonance absorption is demonstrated in the inset of Fig. 7 .

\section{Mean-field model}

A phenomenological approach has an essential limit to describe well the properties of the manganites, but sometimes the phenomenological model allows to provide the understanding of the main features in the behavior of the manganites. We use a simplest mean-field approximation, which reproduces qualitatively 
the behavior of two-phase system at change of the external influences: temperature, magnetic field, and magnetic resonance. The same model was first applied to manganites by Jaime and co-workers [11]. Strong electron-phonon interaction is the basis of the proposed model. Really, it is clear now that the Jahn-Teller effect in $\mathrm{Mn}^{3+}$ plays essential role for physics of the manganites [1]. Strong electron-phonon coupling leads to the small polaron formation at $T>T_{\mathrm{c}}$, but below $T_{\mathrm{c}}$ double exchange results in dissolving of the polarons into band electrons.

Near $T_{\mathrm{c}}$ the free-energy functional can be written in the form of expansion in powers of two order parameters. The relative magnetization $m=m(T, H)$ is the preliminary order parameter and we propose that the relative concentration of the ferromagnetic phase $Q=Q(T, H)$ is the secondary order parameter driven by the $m$. If really, the transition from polaronic to metallic conductivity relates to strong electron-phonon coupling, so, in our phenomenological model, we can use expansion of the free energy in power $m$ and $Q_{\mathrm{fm}}$ in a conventional form for ferromagnet with magneto-elastic coupling, i.e.,

$$
F=\frac{1}{2} a m^{2}+\frac{1}{4} b m^{4}+\frac{1}{2} \alpha Q^{2}+\frac{1}{4} \beta Q^{4}+\chi m^{2} Q-M_{0} m_{z} H .
$$

Here term $\chi \mathrm{m}^{2} Q$ is the simplest form of the magneto-elastic coupling, which is assumed by symmetry of the cubic lattice, the last term describes Zeeman energy, $M_{0}$ is saturation magnetization, and we can consider that $\boldsymbol{m} \| \boldsymbol{H}$ at thermodynamic equilibrium. Minimizing $F$ with respect to both parameters and using some generalization, we can obtain the following simultaneous equations:

$$
\begin{aligned}
& m=B_{S}\left\{\frac{3 S T_{\mathrm{c}}^{*}}{(S+1) T}\left[(1+\chi Q) m+\frac{g \mu_{\mathrm{B}}(S+1)}{3 k_{\mathrm{B}} T_{\mathrm{c}}} H\right]\right\}, \\
& Q=\frac{\chi}{\alpha} m^{2} .
\end{aligned}
$$

Here it is necessary to take account that $T_{c}^{*}$ is the temperature of the magnetic phase transition at $\chi=0$. Solving simultaneously these equations we can obtain dependences of the order parameters versus temperature and magnetic field. Figure 8 shows the dependence of the $Q(H)$. It is seen that for $\alpha=0.18, \chi=$ $0.28, T_{\mathrm{c}}=210, S=3 / 2$ satisfactory fit takes place between this dependence and the one obtained from experimental data in the framework of the effective-medium approximation (Fig. 7).

We can analyze the change of $R$ under magnetic resonance using assumption that the observed effect is a direct consequence of the phase separation picture. In this case, the free energies in PM (with polaronic conductivity) and FM (with metallic conductivity) states are nearly equal, and a small local perturbation of one of the subsystems can result in the change of a thermodynamic equilibrium in the mixed state. Near a percolation threshold for the metallic state this situation can imply the visible change of conductivity in the sample. Since the conditions of the excitation of the resonance microwave absorption are different for PM and FM phases at scanning of the magnetic field the local perturbation of either one 


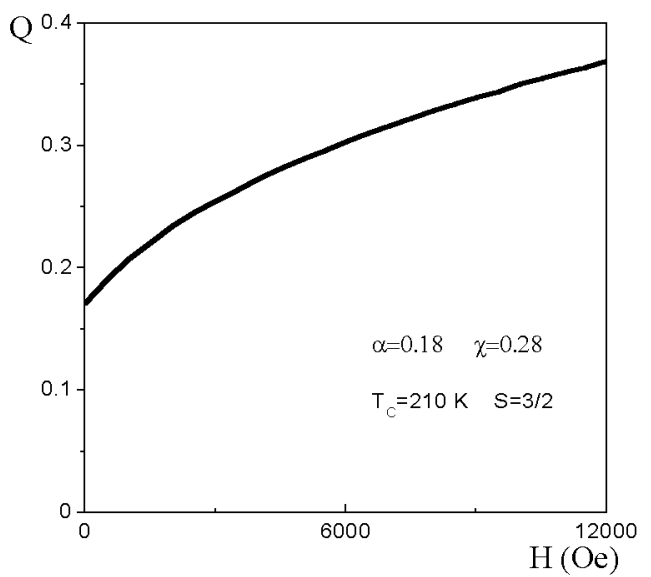

Fig. 8. The magnetic field dependence of concentration of the ferromagnetic phase in the sample. The dependence is calculated in the framework of mean-field model.

or other phase takes place. As a result, the phase equilibrium in the system is changed either in one or other direction, and we observe the increase or decrease in the sample conductivity.

Using simplest phenomenological model proposed above we try to discuss possible mechanisms of change of the phase state under magnetic resonance. We consider separately the situations originating from magnetic resonance absorption in the FM and PM subsystems of the sample. Really, different behavior of the conductivity under PM and FM resonance (for example, see Fig. 3) confirms propriety of such approach.

Under FM resonance the increase in the microwave power results in the decrease in magnetization projection onto a magnetic field direction $m_{z}$. In our model it results in the decrease in the Zeeman energy in the free energy expression (6). To explain the change of $C_{\mathrm{fm}}$ induced by the magnetic resonance absorption in $\mathrm{FM}$ phase (the inset of Fig. 6) the change of the projection $\Delta m_{z}=\Delta M_{z} / M_{0}$ should be $\approx 10^{-3}$. This order of magnitude quite conforms with $\Delta m_{z}$ which takes place under magnetic resonance for the microwave power applied.

It is clear that the change of the conductivity induced by resonance absorption in PM regions should be considered in the framework of other mechanism. The simplest variant is to propose dependence of $\chi$, which determines the coupling of the order parameters, on microwave power absorbed by the PM subsystem. Increase in $\chi$ by $0.07 \%$ allows to explain the change of the concentration of the coexisting phase and relative change of the conductivity under magnetic resonance in PM regions. We can suggest the following qualitative mechanism explaining such increase in $\chi$. Double exchange coupling, which defines magnetic behavior, implies that magnetic ordering results in increase in a kinetic energy of the electrons. According to this an effective value of the electron-phonon coupling decreases. On the contrary, under the magnetic resonance in PM phase a proba- 
bility of an electron scattering by precessed localized magnetic moment of Mn ions grows, this decreases the kinetic energy of the carriers and restores the effective electron-phonon coupling value.

The limitation of $A_{1}$ and $A_{2}$ (see Fig. 4) with dc value increase can be apparently attributed to the electron scattering process, too. Here we should take into account that this process results in intensification of a spin relaxation channel and, as consequence, the amplitude of the precession can be reduced both in PM and FM phases at fixed microwave power.

\section{Conclusion}

In summary, the results shown above provide one more experimental evidence for phase separation in the manganites. The magnetic resonance methods including both the conventional technique, the scheme of spectrometer with the microwave frequency tuning and the method with combined influence of the microwave irradiation and $\mathrm{dc}$ are found to be the powerful instruments for the probing the magnetic inhomogeneity in the manganite single crystals.

\section{Acknowledgments}

This work was supported by KRSF-RFBR "Enisey 2002" (grant No. 02-02-97702) and Program of Department of Physics Sciences RAS "Spin Dependent Effects in Solids and Spintronics".

\section{References}

[1] E. Dagotto, T. Hotta, A. Moreo, Phys. Rep. 344, 1 (2001).

[2] J.B. Goodenough, Aust. J. Phys. 52, 155 (1999).

[3] A.K. Srivastava, C.M. Srivastava, R. Mahesh, C.N.R. Rao, Solid State Commun. 99, 161 (1996).

[4] S.L. Yuan, J.Q. Li, Y.P. Yang, X.Y. Zeng, G. Li, F. Tu, G.Q. Zhang, C.Q. Tang, S.Z. Jin, Phys. Rev. B 62, 5313 (2000).

[5] V.A. Atsarkin, V.V. Demidov, G.A. Vasneva, K. Conder, Phys. Rev. B 63, 092405 (2001).

[6] A.I. Shames, E. Rozenberg, W.H. McCarroll, M. Greenblatt, G. Gorodetsky, Phys. Rev. B 64, 172401 (2001).

[7] G.A. Petrakovskii, N.V. Volkov, V.N. Vasil'ev, K.A. Sablina, JETP Lett. 71, 144 (2000)

[8] N.V. Volkov, G.A. Petrakovskii, V.N. Vasiliev, D.A. Velikanov, K.A. Sablina, K.G. Patrin, Physica B 324, 254 (2002).

[9] M. Salamon, M. Jaime, Rev. Mod. Phys. 73, 583 (2001).

[10] A.N. Lagarkov, A.K. Sarychev, Phys. Rev. B 53, 6318 (1996).

[11] M. Jaime, P. Lin, S.H. Chun, M.B. Salamon, P. Dorsey, M. Rubinstein, Phys. Rev. $B$ 60, 1028 (1999). 